\title{
T cell inhibition by pogostone from Pogostemon cablin (Blanco) Benth: In vitro and in vivo immunosuppressive analysis
}

\author{
JIYAN SU ${ }^{1,2^{*}}$, JINGJIN HE $^{3 *}$, ZIREN SU ${ }^{2,4}$, LIAN ZHOU ${ }^{5}$, YAOYING ZENG ${ }^{6}$, XIAOPING LAI ${ }^{2,4}$ and YUCUI LI $^{2}$ \\ ${ }^{1}$ State Key Laboratory of Applied Microbiology Southern China, \\ Guangdong Provincial Key Laboratory of Microbial Culture Collection and Application, Guangdong Institute of \\ Microbiology, Guangzhou, Guangdong 510070; ${ }^{2}$ Guangdong Provincial Key Laboratory of New Drug Development and \\ Research of Chinese Medicine, Guangzhou University of Chinese Medicine, Guangzhou, Guangdong 510006; \\ ${ }^{3}$ Cancer Research Institute, Southern Medical University, Guangzhou, Guangdong 510515; ${ }^{4}$ Dongguan Mathematical \\ Engineering Academy of Chinese Medicine, Guangzhou University of Chinese Medicine, Dongguan, Guangdong 523000; \\ ${ }^{5}$ School of Chinese Materia Medica, Guangzhou University of Chinese Medicine, Guangzhou, Guangdong 510006; \\ ${ }^{6}$ Institute of Tissue Transplantation and Immunology, Jinan University, Guangzhou, Guangdong 523000, P.R. China
}

Received October 29, 2016; Accepted June 9, 2017

DOI: $10.3892 / \mathrm{mmr} .2017 .7147$

\begin{abstract}
Various plant-derived compounds exhibit immunosuppressive activity in pre-clinical investigations, suggesting that they may serve as natural alternatives for the prevention of inflammatory disorders and autoimmune diseases. The aim of the current study was to explore the immunosuppressive potential of pogostone (PO) derived from Pogostemon cablin (Blanco) Benth. Carboxyfluorescein diacetate succinimidyl ester-labeled cell tracking demonstrated that PO $(20-80 \mu \mathrm{M})$ inhibited Concanavalin A (ConA)-stimulated lymphocyte proliferation, which was mediated by $G_{0} / G_{1}$ phase arrest and accompanied by significant decreases in the expression of CD69 (early-stage activation marker) and CD25 (mid-stage activation marker) in T cells, as indicated by flow cytometry analysis. Furthermore, the proliferation blocking ability of PO $(5-80 \mu \mathrm{M})$ was not associated with cytotoxicity in normal lymphocytes or apoptosis in ConA-stimulated lymphocytes. The inflammatory cytokine profile determination using a cytometric beads assay revealed that PO inhibited release of anti-inflammatory interleukin (IL)-10 and pro-inflammatory IL- 6 from the stimulated lymphocytes. Furthermore, PO $(10,20$ or $40 \mathrm{mg} / \mathrm{kg})$ ameliorated the T-cell mediated delayed
\end{abstract}

Correspondence to: Professor Xiaoping Lai or Dr Yucui Li, Guangdong Provincial Key Laboratory of New Drug Development and Research of Chinese Medicine, Guangzhou University of Chinese Medicine, 232 East Waihuan Road, Higher Education Mega Center of Guangzhou, Guangzhou, Guangdong 510006, P.R. China E-mail:1xp88@gzucm.edu.cn

E-mail: liyucui@gzucm.edu.cn

${ }^{*}$ Contributed equally

Key words: pogostone, T cell, inactivation, immunosuppression type hypersensitivity response in Balb/c mice by reducing leukocyte infiltration and tissue edema, providing a further validation of the direct immunosuppressive activity of PO. Together, the present data suggest that PO would suppress $\mathrm{T}$ cell response via a direct non-cytotoxic inactivation at the early stage, accompanied by regulation of the inflammatory cytokine profile, which highlights clinical implications for treatment of immune-based disorders.

\section{Introduction}

T cells are a principle component of the cell-mediated immune system, which has a key role in the generation of an efficient, yet controlled, adaptive immune response, particularly following potential threats from pathogens or neoplasia (1). However, excessive activation or proliferation of T cells is a major cause of a multitude of inflammatory disorders, including allergies and transplantation rejection (2). Furthermore, certain life-threatening autoimmune diseases are associated with the over-activation of $\mathrm{T}$ cell, including systemic lupus erythematosus and inflammatory bowel diseases $(3,4)$. Thus, inhibitors targeting $\mathrm{T}$ cell or drugs that neutralize $\mathrm{T}$ cell-derived cytokines are considered as powerful reagents for treatment of patients with the aforementioned inflammatory diseases. In addition to the regents with direct cytotoxic activity against T lymphocytes (such as methylprednisolone and azathioprine), clinical immunosuppressants employ strategies that focus on activation inhibition (such as cyclosporine), proliferation blocking (such as rapamycin,) and cytokine neutralization (such as infliximab), all of which are prerequisites that enable T lymphocytes to drive adaptive immunity.

There are three main signaling pathways that are utilized by immunosuppressants. The first is activation of $\mathrm{T}$ cell receptor (TCR)/CD3-mediated nuclear factor of activated $\mathrm{T}$ cells, which is initiated by antigen presentation on a major histocompatibility complex (MHC) protein, against which cyclosporine and FK506 are the representative inhibitors. The 
second is B7/CD28 co-stimulation that facilitates TCR-MHC complex synapsing. The third pathway is interleukin (IL)-2/IL receptor $2 \alpha$ chain (CD25) signaling, which affects cell cycle control via regulation of cyclin-dependent kinases and cyclins through the Janus kinase (JAK)/phosphoinositide 3-kinase/mechanistic target of rapamycin (mTOR) pathway (5). However, immunosuppressants used in the clinic have various deficiencies or adverse effects. For example, cyclosporine acts as an immunosuppressant by interfering with the activity and growth of T cells, and was listed as one of the most important medications to prevent rejection of transplants in the World Health Organization Model List of Essential Medicines in 2015 (6); however, cyclosporine has also been reported to be nephrotoxic, neurotoxic and to increase the risk of squamous cell carcinoma, infections and hypertension (7). Rapamycin is a non-depleting immunosuppressant used to prevent rejection in organ transplantation, with its activity attributed to anti-proliferative properties via mTOR inhibition in lymphocytes. However, rapamycin may induce systemic non-nephrological side effects, including pulmonary toxicity, hematopoietic adverse effects and metabolic disorders, with the majority of the adverse effects linked to the dosage. It is extremely important for clinicians to rapidly recognize the adverse effects in order to reduce the dosage or discontinue mTOR inhibitor treatment, and avoid the onset and development of severe clinical complications (8).

There is growing interest in using herbal medicines as immunoregulators to modulate the complex immune system, preventing inflammation and the development of autoimmune diseases. There are various plant-derived compounds (curcumin, resveratrol, epigallocatechol-3-gallate, quercetin, colchicine, capsaicin, andrographolide and genistein) that exhibit promising effects on innate and adaptive immune functions in pre-clinical investigations, which may elucidate their clinical potential (9). Pogostemon cablin (Blanco) Benth. is a valuable medicinal and aromatic plant used for many years in southeast Asia, particularly in China, India and Philippines. As a traditional Chinese herbal medicine, the dried aerial part of $P$. cablin is used to treat sunstroke-associated headache, fever, emesis and even coma. There are numerous studies demonstrating that $P$. cablin has a variety of pharmacological activities, including anti-inflammation and analgesia (10), immunoregulation (11) and anti-emesis (12). Pogostone (PO; Fig. 1A) is a chemical marker of P. cablin, and it has been reported to be responsible for the majority of the activities of $P$. cablin, including anti-bacterial (13) and anti-fungal (14) effects. In the present study, a Concanavalin A (ConA)-stimulation model in vitro and a $\mathrm{T}$ cell-mediated delayed type hypersensitivity (DTH) model in vivo were used to determine the potential of $\mathrm{PO}$ as an inhibitor of $\mathrm{T}$ cell responses.

\section{Materials and methods}

Animals. A total of $50 \mathrm{Balb} / \mathrm{c}$ mice (8-week-old, male, 18-22 g) were provided by the Laboratory Animal Center of Guangzhou University of Chinese Medicine (Guangzhou, China). The mice were acclimatized and raised under specific pathogen-free conditions at temperature of $24 \pm 1^{\circ} \mathrm{C}$, humidity of $40-80 \%$ and a $12 \mathrm{~h}$-light/12 h-dark cycle. All experiments were performed according to the National Institutes of Health Guidelines for Care and Use of Laboratory Animals (15) and were approved by the Bioethics Committee of Guangzhou University of Chinese Medicine.

Materials. PO (purity, $\geq 98 \%$ ) was isolated from P. cablin as described previously (13). The aerial parts of $P$. cablin were collected in October 2010 in Maoming (Guangdong, China). The species was authenticated by Professor Xiaoping Lai at the Guangdong Provincial Key Laboratory of New Drug Development and Research of Chinese Medicine, Guangzhou University of Chinese Medicine where a voucher specimen (no. 101009) was deposited.

Briefly, dried aerial parts of P. cablin $(3.8 \mathrm{~kg}$ ) were extracted by distillation, and the extract was dissolved in ethyl acetate $(50 \mathrm{ml})$. The obtained ethyl acetate fraction was extracted using $4 \% \mathrm{NaOH}$ five times, and the aqueous fractions were pooled. Following adjusting to $\mathrm{pH} 2.0$ using $10 \% \mathrm{HCl}$, the aqueous fraction was extracted by the ethyl acetate three times in a total volume of $200 \mathrm{ml}$. The pooled ethyl acetate extracts were washed with $400 \mathrm{ml}$ water, dried by evaporation and finally crystallized in n-hexane to obtain white PO crystals (178.6 mg; yield $0.0047 \%$ ). The chemical structure and purity of PO was identified and determined as reported previously $(16,17)$.

ConA type IV, propidium iodidedimethyl (PI), carboxyfluorescein diacetate succinimidyl ester (CFSE) were purchased from Sigma-Aldrich (Merck KGaA, Darmstadt, Germany). Cyclophosphamide (CTX) was provided by Tonghua Maoxiang Pharmaceutical Co., Ltd. (Jilin, China). Indomethacin was obtained from Guangdong Huanan Pharmaceutical Co., Ltd. (Guangdong, China). Phycoerythrin-anti-mouse CD3 (cat. no. 553240), fluorescein isothiocyanate (FITC)-anti-mouse CD69 (cat. no. 557392), FITC-anti-mouse CD25 (cat. no. 553071), and FITC-anti-mouse transferrin receptor (CD71; cat. no. 561936) and a FITC-Annexin V Apoptosis Detection kit (cat. no. 556547) were purchased from BD Pharmingen (San Jose, CA, USA). Cell Counting Kit-8 (CCK-8) was from Dojindo Molecular Technologies, Inc. (Kumamoto, Japan). Mouse Inflammation Cytometric Bead Array (CBA) kit was purchased from BD Biosciences (San Jose, CA, USA).

Cell preparation. Balb/c mice were sacrificed, and lymph nodes in bilateral jaws, armpit, clavicle, groin and mesentery were aseptically isolated and ground in cold phosphate-buffered saline (PBS) by passing through a $70 \mu \mathrm{m}$ strainer. Lymphocytes were collected and washed twice with cold PBS by centrifugation at $250 \mathrm{x}$ g at $4^{\circ} \mathrm{C}$ for $5 \mathrm{~min}$. The collected cells were resuspended in complete RPMI-1640 medium (Gibco; Thermo Fisher Scientific, Inc.) containing 10\% heat-inactivated fetal bovine serum (FBS; Gibco; Thermo Fisher Scientific, Inc.), and $100 \mathrm{IU} / \mathrm{ml}$ penicillin and streptomycin, except for in the of proliferation assay. Cell viability was determined by trypan-blue dye exclusion with $0.4 \%$ trypan blue for $2 \mathrm{~min}$, and cell viability was $>95 \%$ in all cases. Cells were cultured in a humidified atmosphere of $5 \% \mathrm{CO}_{2}$ at $37^{\circ} \mathrm{C}$.

Cytotoxicity assay. Lymphocytes (190 $\mu \mathrm{l})$ were seeded at a density of $3 \times 10^{6}$ cells $/ \mathrm{ml}$ in 96 -well plates (triplicate wells in each group), and treated with $10 \mu \mathrm{l}$ PO in complete RPMI-1640 medium at multiple concentrations $(5,10,20,40$ and $80 \mu \mathrm{M}$, 
A<smiles>[AsH2]</smiles>

C

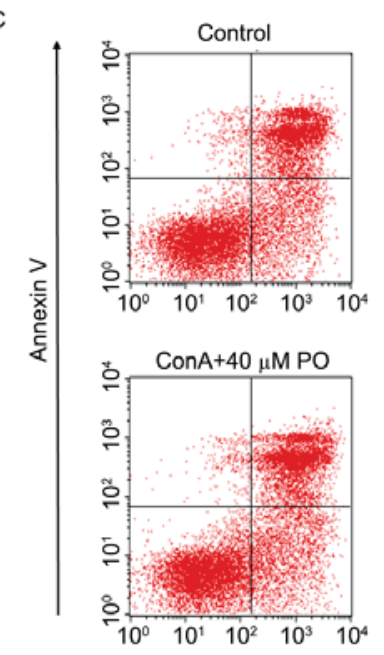

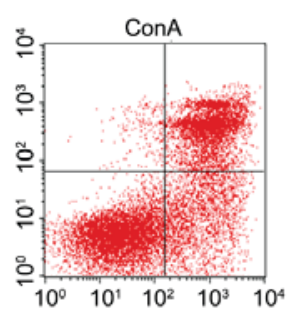

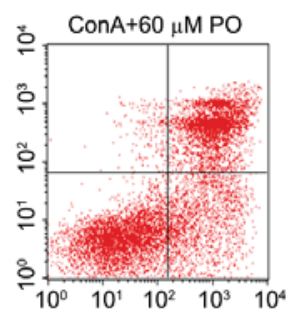

B

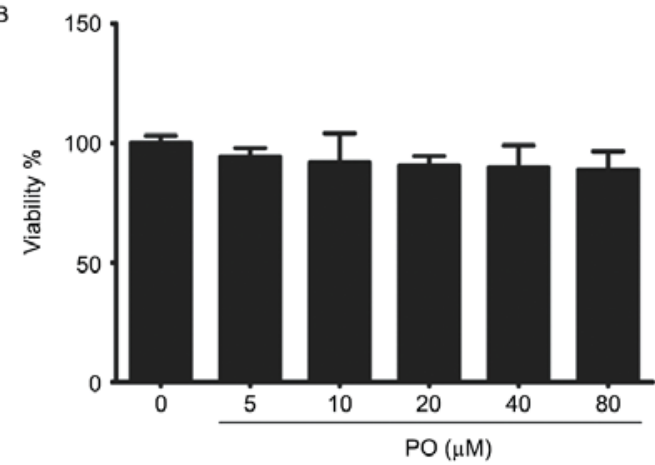

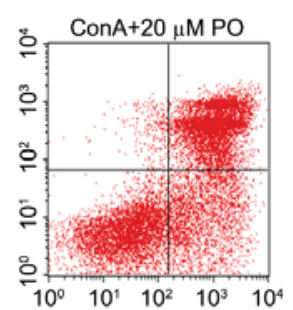
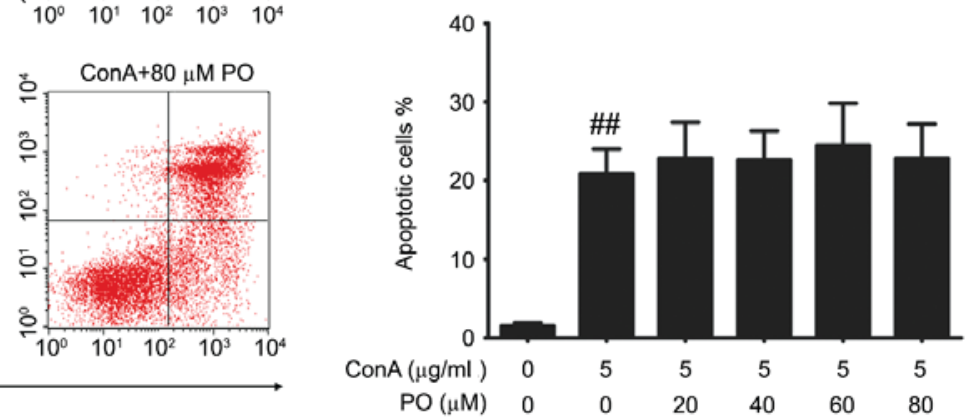

Figure 1. Viability and apoptosis analysis. (A) Structure of PO; chemical name, 4-hydroxy-6-methyl-3-(4-methylpentanoyl)-2 $\mathrm{H}$-pyran-2-one $\left(\mathrm{C}_{12} \mathrm{H}_{16} \mathrm{O}_{4}\right)$; and molecular weight, 224. (B) Viability of splenotyes following treatment with PO for $48 \mathrm{~h}$. (C) Apoptosis analysis of the 24 h-ConA-stimulated splenocytes. All data were statistically analyzed and presented in column charts. Values are presented as the mean \pm standard deviation ( $\mathrm{n}=3$ ). ${ }^{\# \#} \mathrm{P}<0.01$ vs. control group $\mathrm{PO}$, pogostone; ConA, Concanavalin A; PI, propidium iodidedimethyl.

with no more than $0.1 \%$ dimethyl sulfoxide). After $48 \mathrm{~h}, 80 \mu \mathrm{l}$ supernatant were removed and $10 \mu \mathrm{lCCK}-8$ was added to each well and the cells were further incubated for another $4 \mathrm{~h}$. The optical density was measured at $490 \mathrm{~nm}$ using a microplate reader.

Proliferation assay. For proliferation assay, the isolated lymphocytes were resuspended in PBS with 5\% FBS, counted and incubated with $2.5 \mu \mathrm{M} \mathrm{CFSE}$ at a density of $5 \times 10^{6}$ cells $/ \mathrm{ml}$ for $8 \mathrm{~min}$ in the dark at room temperature, washed twice with $5 \% \mathrm{FBS} / \mathrm{PBS}$ and finally resuspended in complete medium. CFSE-labeled cells were seeded at a density of $3 \times 10^{6} \mathrm{cell} / \mathrm{s} / \mathrm{ml}$ in 96-well plates (triplicate wells), and subjected to a 4-h pretreatment of PO (20, 40, 60 and $80 \mu \mathrm{M})$. Subsequently, $5 \mu \mathrm{g} / \mathrm{ml}$ ConA were added into the mixture to stimulate the lymphocyte for another $72 \mathrm{~h}$. Finally, cells were harvested, washed with PBS, and resuspended in $2 \%$ paraformaldehyde. The division profiles of responder cells were analyzed based on the CFSE content with FACS Calibur flow cytometer (BD Biosciences) and data were analyzed using CellQuest Pro 6.0 software (BD Biosciences).

Apoptosis analysis. Lymphocytes were seeded into 96-well plates (triplicate wells) at a density of $3 \times 10^{6}$ cells $/ \mathrm{ml}$ in $100 \mu \mathrm{l}$ and pre-treated with $100 \mu \mathrm{l} \mathrm{PO}(20,40,60$ and $80 \mu \mathrm{M})$ for $4 \mathrm{~h}$ following by a stimulation with ConA $(5 \mu \mathrm{g} / \mathrm{ml})$ for another 24 h. Cells were harvested, washed once with PBS, and stained with Annexin V-FITC and PI in $500 \mu \mathrm{l}$ binding buffer for $30 \mathrm{~min}$ in dark at room temperature according to the manufacturers' instruction (BD Pharmingen). Characteristics of the cells were acquired and analyzed using a FACS Calibur flow cytometer and CellQuest software.

Cell cycle assay. Following PO pre-treatment (20, 40, 60 and $80 \mu \mathrm{M})$ and ConA stimulation $(5 \mu \mathrm{g} / \mathrm{ml})$ for $48 \mathrm{~h}$, cells were fixed and permeabilized in cold $70 \%$ ethanol $\left(-20^{\circ} \mathrm{C}\right)$ containing $10 \%$ FBS for $30 \mathrm{~min}$. Following two cold PBS washes, cells were labeled with PI staining solution $(50 \mu \mathrm{g} / \mathrm{ml})$ in the dark for $5 \mathrm{~min}$ at room temperature before analysis with the flow cytometer. Cell cycle propagation, as determined by DNA ploidy, was assessed on the basis of PI incorporation using the FACS Calibur flow cytometer, and data were analyzed by ModFit LT 3.1 software (Verity Software House, Inc., Topsham, ME, USA).

Flow cytometry analysis of CD69, CD25 and CD71 expression. After PO treatment $(20,40,60$ and $80 \mu \mathrm{M})$ for $4 \mathrm{~h}$, the lymphocytes were stimulated with ConA $(5 \mu \mathrm{g} / \mathrm{ml})$ for 8,16 and $24 \mathrm{~h}$. Cells were harvested and stained with PE-CD3 $(0.125 \mu \mathrm{g} /$ test $)$ plus FITC-CD69 (0.25 $\mu \mathrm{g} /$ test $)$ antibodies for 
the $8 \mathrm{~h}$ samples, PE-CD3 $(0.125 \mu \mathrm{g} /$ test $)$ plus FITC-CD25 $(0.25 \mu \mathrm{g} /$ test $)$ antibodies for the $16 \mathrm{~h}$ samples, and PE-CD3 $(0.125 \mu \mathrm{g} /$ test $)$ plus FITC-CD71 $(0.25 \mu \mathrm{g} /$ test $)$ antibodies for the $24 \mathrm{~h}$ samples on ice for $30 \mathrm{~min}$. Following two washes in PBS, cells were resuspended in $2 \%$ paraformaldehyde and detected using the FACS Calibur flow cytometer. The data was analyzed by CellQuest software.

Determination of inflammation cytokine profiles. Release of cytokines in the supernatant, including IL-6, IL-10, monocyte chemoattractant protein-1 (MCP-1), interferon- $\gamma($ IFN- $\gamma$ ) and tumor necrosis factor (TNF) were measured using the inflammation CBA according to the manufacturer's instruction. Briefly, the obtained lymphocytes were seeded into 96-well plates (triplicate wells) at a density of $3 \times 10^{6}$ cells $/ \mathrm{ml}$ in $100 \mu \mathrm{l}$ and pre-treated with $100 \mu \mathrm{l} \mathrm{PO}(20,40,60$ and $80 \mu \mathrm{M})$ for $4 \mathrm{~h}$ followed by stimulation with ConA $(5 \mu \mathrm{g} / \mathrm{ml})$ for a further $72 \mathrm{~h}$, and then $25 \mu 1$ supernatant was collected and incubated with the mixture of mouse inflammation capture bead suspension and the PE detection reagent for $2 \mathrm{~h}$. Samples were washed with PBS and detected by FACS Calibur flow cytometer, and data was analyzed using BD CBA software. Mouse Inflammation standards provided with the kit were appropriately diluted and used in parallel to samples for preparation of the standard curves.

Dinitrochlorobenzene (DNCB)-induced DTH reaction. Balb/c mice were randomly divided into 6 groups ( $n=6$ per group). Except for mice of the control group, DTH reaction was initiated by smearing $50 \mu 11 \%$ DNCB (dissolved in acetone) on the shaved abdominal skin of the mice, and was elicited 6 days later by smearing $10 \mu 11 \%$ DNCB on both sides of the left ear. On day 1 of immunization, mice were orally administered with either PO $(10,20$ or $40 \mathrm{mg} / \mathrm{kg}$, dissolved in $2 \%$ polysorbate 80 ) or cyclophosphamide (CTX; $37 \mathrm{mg} / \mathrm{kg}$ ) once per day for 7 consecutive days. The normal and model control groups received the same volume of saline. At $24 \mathrm{~h}$ after the second challenge, DTH reaction was measured as indicated by the increased ear patch weight (8-mm punches) between the left and right ear.

The ear patches were then fixed in $4 \%$ formaldehyde $(\mathrm{pH}$ 7.2) at room temperature for 24-48 $\mathrm{h}$. The fixed patches were dehydrated, embedded in paraffin, and sectioned into 3-5 $\mu \mathrm{m}$ slices. The sections were dewaxed in xylene, rehydrated through decreasing concentrations of ethanol, and washed in PBS. Sections were stained with $0.8 \%$ eosin and Mayer's hematoxylin solution at room temperature, for $8 \mathrm{~min}$ and $15 \mathrm{~min}$, respectively. Following staining, sections were dehydrated through increasing concentrations of ethanol and xylene. Five high-power fields (magnification, x400) were randomly selected in each slide with BX61 imaging system (Olympus Corp., Tokyo, Japan) to assess the histological alterations induced by the DTH reaction.

Statistical analysis. A minimum of 10,000 cells were assessed in flow cytometry analysis. All experiments were performed at least in triplicate, and the results are expressed as the mean \pm standard deviation. Statistical analysis was performed by one-way analysis of variance using SPSS 18.0 (SPSS, Inc., Chicago, IL, USA). A post hoc least significant difference test was applied for difference analysis under homogeneity of variance, otherwise, a Dunnett's test was applied. $\mathrm{P}<0.05$ was considered to indicate a statistically significant difference.

\section{Results}

PO has limited influence on cell viability and apoptosis. As presented in Fig. 1B, viabilities of the treated lymphocytes were all $>70 \%$ after a $48 \mathrm{~h}$-incubation with $\mathrm{PO}(5-80 \mu \mathrm{M})$ compared with the control group, indicating that low concentrations of PO had no toxicity on lymphocytes (Fig. 1B). Additionally, Annexin V-PI staining indicated that the $24 \mathrm{~h}$ ConA stimulation marginally induced apoptosis in lymphocytes, which may be as a consequence of activation-induced cell death, while no increase in apoptosis was observed in the PO-treated lymphocytes (Fig. 1C and D). Together these results suggested that PO has little influence on cell viability and apoptosis.

PO inhibited ConA-stimulated lymphocyte proliferation via $G_{0} / G_{I}$ phase arrest. To examine the proliferation inhibition effect of PO, lymphocytes were stained with CFSE to detect proliferating cells. Following ConA stimulation, lymphocytes exhibited higher proliferation indicated by the increased percentages of dividing cells compared with untreated cells (Fig. 2). Furthermore, PO decreased the proliferative proportion of total lymphocyte cells following 48 and $72 \mathrm{~h}$ treatment (Fig. 2). Together with data of viability and apoptosis, it can be concluded that PO directly inhibited ConA-stimulated lymphocyte proliferation, but not via effects on apoptosis.

Cell cycle analysis was performed to investigate the direct inhibition of $\mathrm{PO}$ on ConA-mediated proliferation. After ConA stimulation for $48 \mathrm{~h}$, populations of the lymphocytes within $\mathrm{S}$ phase and $\mathrm{G}_{2} / \mathrm{M}$ phase were markedly increased, while PO (20,40, 60 and $80 \mu \mathrm{M})$ prominently reversed this phenomenon. The proportion of the PO-treated lymphocytes in $\mathrm{G}_{0} / \mathrm{G}_{1}$ phase significantly increased as the PO dose increased, and simultaneously those in $\mathrm{S}$ and $\mathrm{G}_{2} / \mathrm{M}$ declined (Fig. 3), suggesting that the PO inhibition of ConA-stimulated proliferation may be attributed to an arrest in $\mathrm{G}_{0} / \mathrm{G}_{1}$ phase.

PO suppressed $T$ cell activation mediated by ConA. Lymphocyte activation promotes the expression of several cell surface proteins. In the present study, it was demonstrated that CD69 (indicating the early-stage activation), CD25 (mid-stage) and CD71 (late-stage) were all highly expressed in T cells $\left(\mathrm{CD}^{+}\right)$under ConA stimulation (Fig. 4). By contrast, PO significantly suppressed the early- and mid-stage activation as characterized by the decreased expressions of CD25 and CD69. However, PO did not affect the expression of CD71, suggesting that the late-stage activation was not influenced by PO (Fig. 4).

Effect of $\mathrm{PO}$ on the inflammatory cytokine profile. As demonstrated in Fig. 5, levels of IL-6, IL-10, IFN- $\gamma$ and TNF- $\alpha$ increased significantly following ConA stimulation for $72 \mathrm{~h}$ $(\mathrm{P}<0.01)$, whereas that of MCP-1 was suppressed. PO had varying impacts on these cytokines. $\mathrm{PO}$ significantly inhibited the ConA-induced release of anti-inflammatory IL-10 and pro-inflammatory IL-6 $(\mathrm{P}<0.01)$, but did not effect that of IFN- $\gamma$ or TNF- $\alpha(\mathrm{P}>0.01)$. Notably, $\mathrm{PO}$ at 20,40 and $60 \mu \mathrm{M}$ 


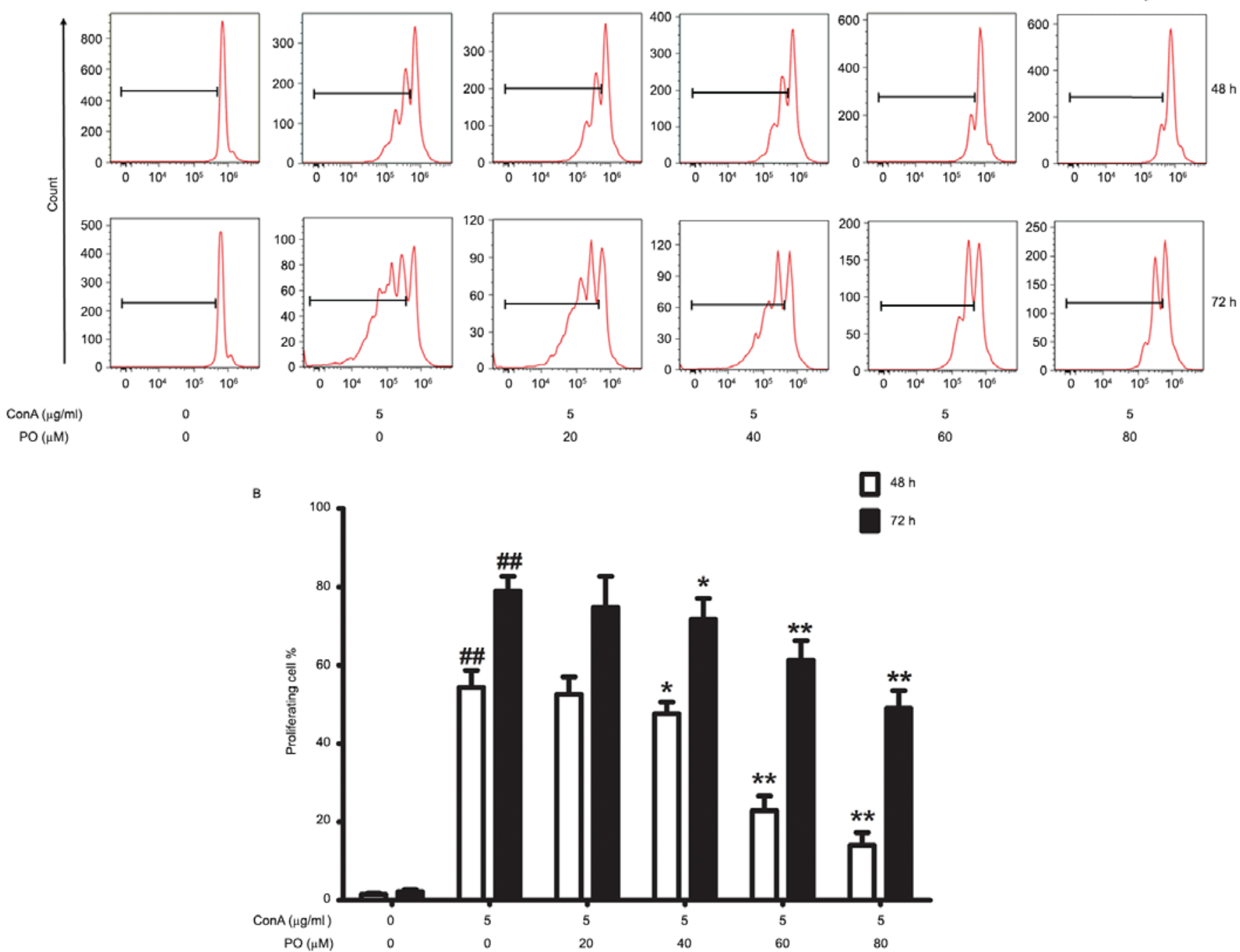

Figure 2. Proliferation analysis of the 72 h-ConA-stimulated lymphocytes. (A) Representative plots from each group demonstrating dividing cell proportions and (B) the proliferating percentage for CFSE staining in response to ConA with or without PO treatment for $72 \mathrm{~h}$. All data were statistically analyzed and presented in column charts. Values are presented the mean \pm standard deviation $(\mathrm{n}=3) .{ }^{\#} \mathrm{P}<0.05$ and ${ }^{\# /} \mathrm{P}<0.01$ vs. control group; ${ }^{*} \mathrm{P}<0.05$ and ${ }^{* *} \mathrm{P}<0.01$ vs. $\mathrm{Con} \mathrm{A}$ group. CFSE, carboxyfluorescein diacetate succinimidyl ester; ConA, Concanavalin A; PO, pogostone.

exhibited similar suppressions on IL-10 and IL-6, whereas $80 \mu \mathrm{M}$ PO did not alter IL-10 and IL-6 levels. Regarding MCP-1, only $20 \mu \mathrm{M}$ PO made an evident increase in the levels following ConA stimulation.

Effect of PO on DNCB-induced DTH response. A DNCB-induced DTH model was used to confirm the immunosuppressive activity of $\mathrm{PO}$ in vivo. Under DNCB elicitation, the left ears of the model group mice were observably more edematous and weighed more than those of the control group (Fig. 6A; P<0.01). By contrast, the immunosuppressant CTX and $40 \mathrm{mg} / \mathrm{kg}$ PO evidently relieved the DNCB-induced DTH response by reducing the ear swelling compared with the model group $(\mathrm{P}<0.01)$, whereas 10 or $20 \mathrm{mg} / \mathrm{kg}$ PO exhibited no effect on the DTH response.

More details of the effect of PO on the DTH response were displayed by HE staining (Fig. 6B). Corresponding to the results of ear patch measurement, DNCB elicitation caused evident tissue hypertrophy and edema in the ears of model group, in which monocytes, eosinophils and neutrophils were markedly increased with evident infiltration into perivascular connective tissue. By contrast, CTX apparently relieved the tissue swellings, and inflammatory cell infiltration. PO only suppressed ear swelling at the $40 \mathrm{mg} / \mathrm{kg}$ dose under DNCB challenge (Fig. 6A); however, HE staining demonstrated that all doses of PO inhibited the cellular immune response by relieving leukocyte infiltration and tissue edema (Fig. 6B).

\section{Discussion}

Various autoimmune diseases and inflammatory disorders are attributed to the hyperactivity of $\mathrm{T}$ cell responses $(3,4)$. Activation and proliferation are the two main prerequisites to initiate the $\mathrm{T}$ cell response against specific antigens, which, therefore, are considered the main targets of most immunosuppressants $(18,19)$. Due to the reported adverse effect from the current clinically used immunosuppressive reagents $(7,20)$, it is an on-going endeavor to identify and develop novel immunosuppressants, particularly from natural products. 

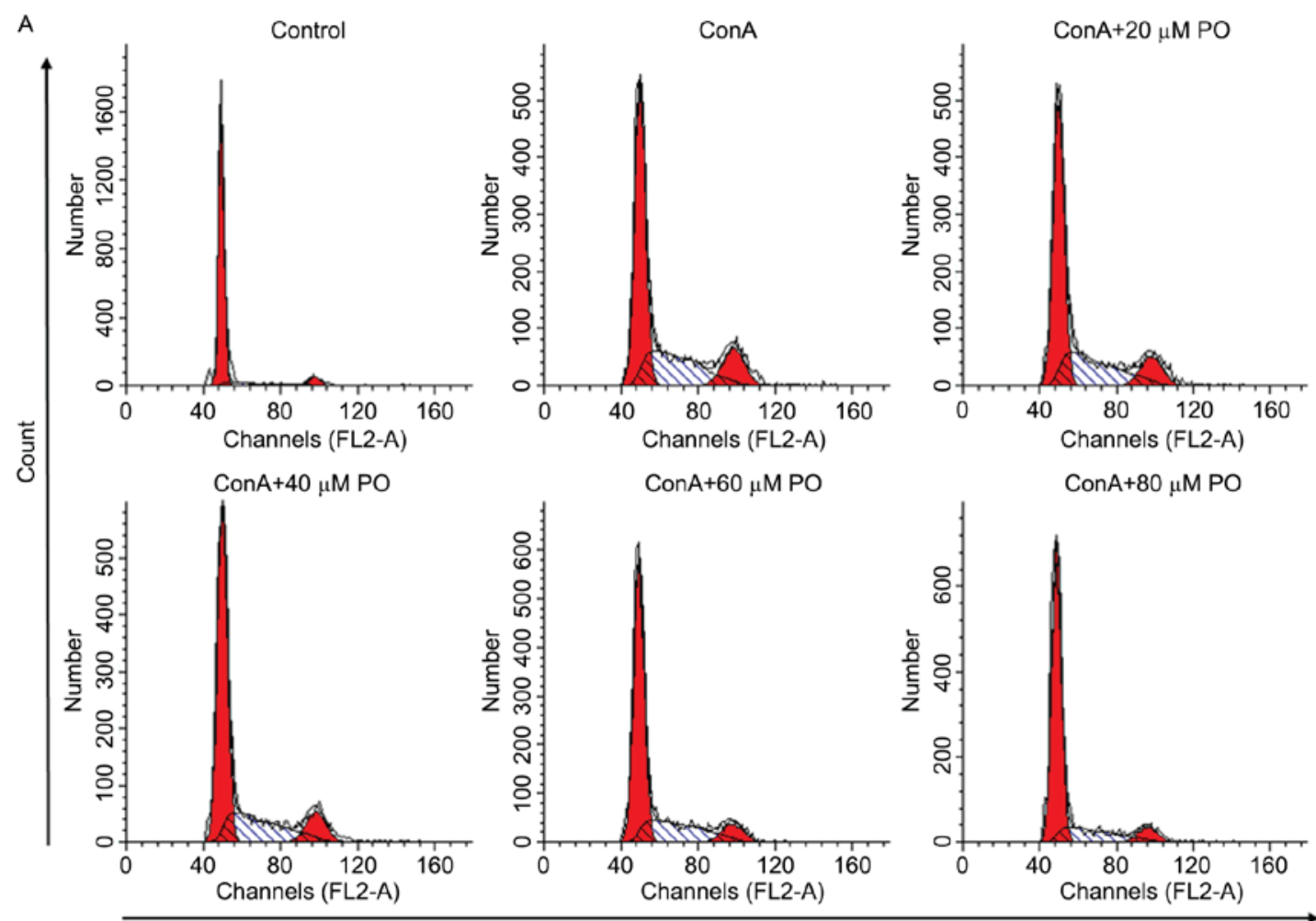

$\mathrm{PI}$

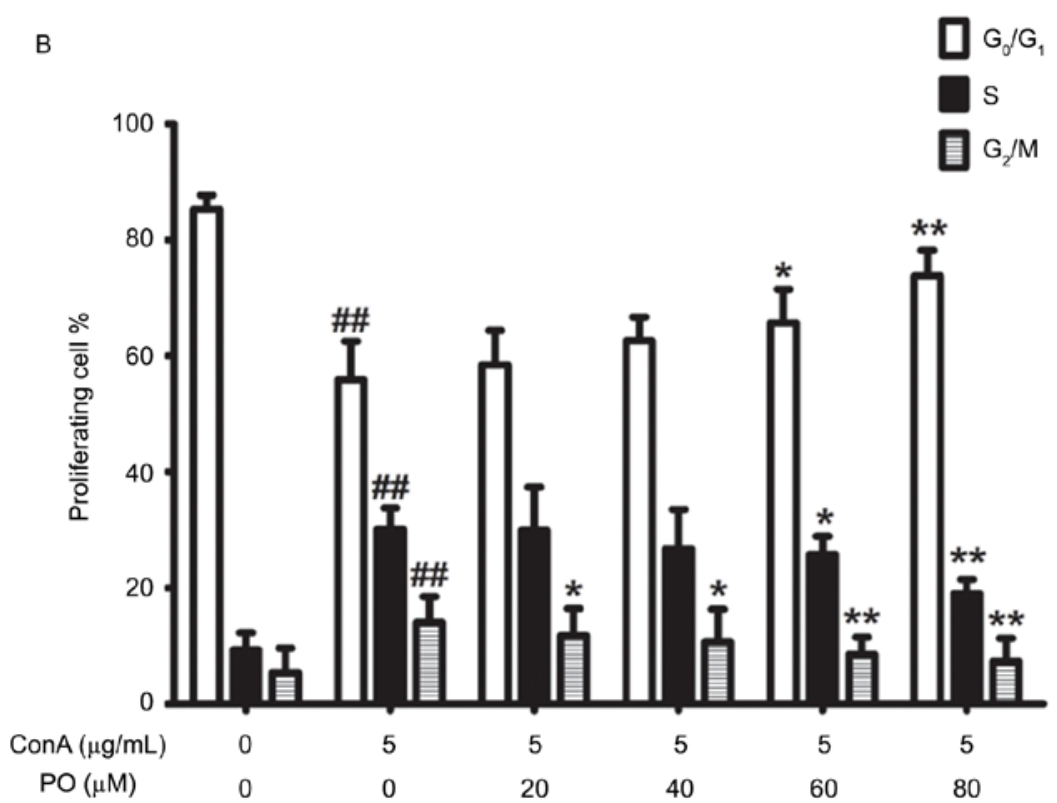

Figure 3. Cell cycle analysis of the 48 h-ConA-stimulated lymphocytes. (A) The cell cycle distribution of lymphocyte was determined using flow cytometry and analyzed by ModFit. (B) All data were statistically analyzed and presented in column charts. Values are presented the mean \pm standard deviation ( $\mathrm{n}=3$ ). ${ }^{\# \#} \mathrm{P}<0.01$ vs. control group; ${ }^{*} \mathrm{P}<0.05$ and ${ }^{* * *} \mathrm{P}<0.01$ vs. Con A group. Con A, Concanavalin A; PO, pogostone; PI, propidium iodidedimethyl.

ConA is a plant mitogen, and is known for its ability to stimulate mouse T-cell subsets giving rise to four functionally distinct $\mathrm{T}$ cell populations, including precursors to suppressor T-cell, hence ConA was employed to study the effect of PO on activated lymphocytes (21). Results of the current study demonstrated that PO significantly reduced lymphocyte proliferation under ConA stimulation, and also arrested the simulated lymphocytes at $\mathrm{G}_{0} / \mathrm{G}_{1}$ phase. Additionally, PO did not induce cytotoxicity or apoptosis in lymphocytes, as indicated by the fact that cell viability and apoptosis of lymphocytes upon ConA stimulation were not changed by PO treatment. As ConA is a non-specific mitogen of $\mathrm{T}$ cell, it is reasonable to speculate that $\mathrm{PO}$ may be a direct inhibitor of $T$ cell proliferation via $G_{0} / G_{1}$ phase arrest. Azathioprine is an immunosuppressant used in organ transplantation and autoimmune diseases; metabolites of azathioprine incorporate into replicating DNA leading to cell cycle arrest, and therefore, strong suppression of bone marrow T cells and B cells. However, one adverse effect of this suppression is apoptosis in $\mathrm{T}$ cells, which may damage the homeostasis of the immune system, resulting in fatigue and anemia (22). By contrast, as 
A
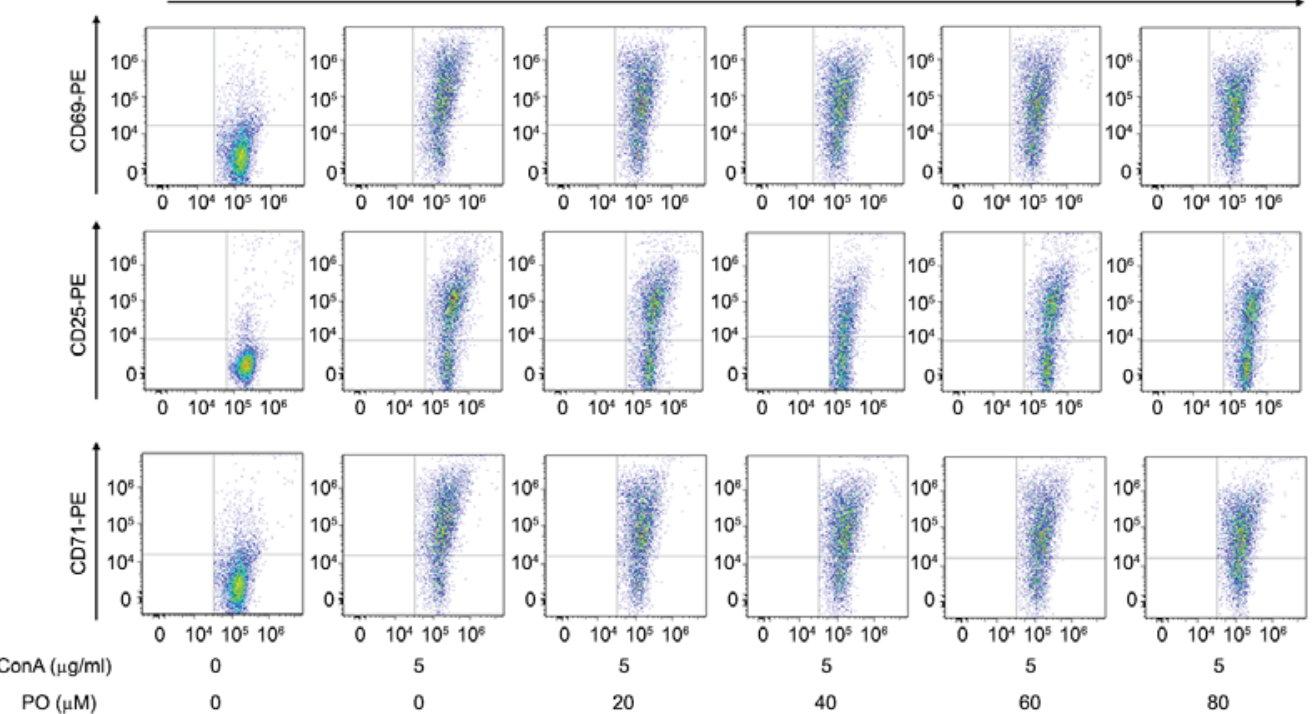

C

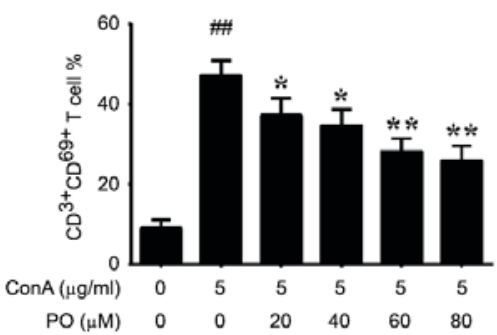

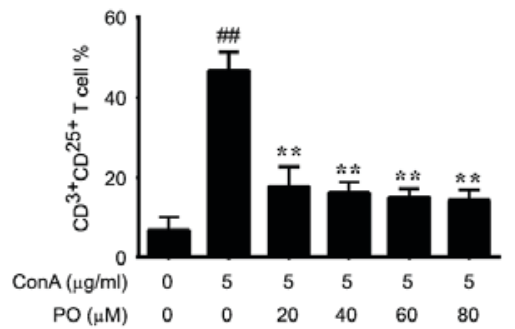

60

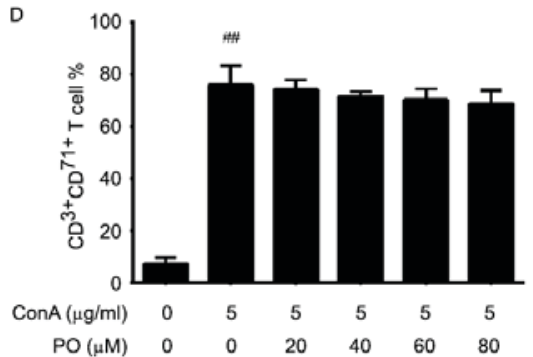

Figure 4. CD69, CD25 and CD71 expression analysis of the ConA-stimulated lymphocytes after 8 h, 16 h, and 24 h, respectively. (A) Typical flow cytometry plots from each group. Percentages of (B) $\mathrm{CD}^{+} \mathrm{CD} 69^{+},(\mathrm{C}) \mathrm{CD}^{+} \mathrm{CD} 25^{+}$and (D) $\mathrm{CD} 3{ }^{+} \mathrm{CD} 71^{+}$cells were statistically analyzed and displayed in column charts Values are presented the mean \pm standard deviation $(\mathrm{n}=3)$. ${ }^{\# /} \mathrm{P}<0.01$ vs. control group; ${ }^{*} \mathrm{P}<0.05$ and ${ }^{* *} \mathrm{P}<0.01$ vs. ConA group. FITC, fluorescein isothiocyanate; PE, phycoerythrin; CD25, IL receptor $2 \alpha$ chain; CD71, transferrin receptor; ConA, Concanavalin A; PO, pogostone; PI, propidium iodidedimethyl.

demonstrated by the present data, PO directly blocked the cell cycle by arresting proliferating lymphocytes in $\mathrm{G}_{0} / \mathrm{G}_{1}$ phase, without inducing cytotoxicity or apoptosis at low concentrations, suggesting that PO may be a potential immunosuppressant alternative without bone marrow suppression effects.

Upregulated expression of membrane proteins on lymphocytes that act as co-stimulatory markers is required for complete lymphocyte activation and effector function. CD69 is the earliest inducible cell surface glycoprotein that is required during lymphoid activation and proliferation of $\mathrm{T}$ lymphocytes and natural killer (NK) cells, in vivo and in vitro. It also functions as a signal-transmitting receptor in NK cells and platelets (23). CD25, the marker for mid-stage activation, is a transmembrane protein present on activated lymphocytes, including $\mathrm{T}$ cell, B cells, some thymocytes and myeloid precursors. CD25 serves as the $\alpha$ chain of the IL-2 receptor to initiate signaling pathways involving JAKs and STATs (24). Consistent with the direct proliferation blocking, PO suppressed ConA-induced upregulation of T cell activation markers, CD69 (early activation) and CD25, but did not affect the expression of CD71 (also known as transferrin receptor), indicating that the immunosuppressive activity of PO is associated with the inactivation of $\mathrm{T}$ cells at the early stage, so as to suppress the subsequent effector functions, including proliferation, cytokine release and differentiation.
Cytokines are critical proteins or compounds secreted by lymphocytes as part of autocrine or paracrine pathways for the purpose of inducing certain cellular processes and signal transduction. Cytokines are classified into several types according to their specific function, including IFNs, ILs, lymphokines, chemokines, colony stimulating factors, TNFs, transforming growth factor- $\beta \mathrm{s}$, growth factors, and appropriate regulation of the cytokine profile is of great importance to maintain a healthy immune system (25); otherwise the immune system can be driven to inflammation or immunodeficiency, resulting in life-threatening physiological responses. IFN- $\gamma$ is a critical cytokine for defenses against viral, certain bacterial and protozoal infections through inhibition of replication (26), and is the pivotal activator of macrophages and inducer of class II MHC expression to stimulate innate and adaptive immunity (27). However, aberrant IFN- $\gamma$ production is associated with a number of auto-inflammatory and autoimmune diseases. IL-6 is a cytokine with pleiotropic influence on antigen-specific immune responses, contributing to host defense via stimulation of acute phase responses, hematopoiesis and immune reactions, while a dysregulated continual synthesis of IL-6 has a pathological effect on chronic inflammation and autoimmunity (28). By contrast, IL-10 is arguably the most potent anti-inflammatory cytokine, which inhibits production of pro-inflammatory cytokines, antigen presentation, and cell proliferation (29); 
A
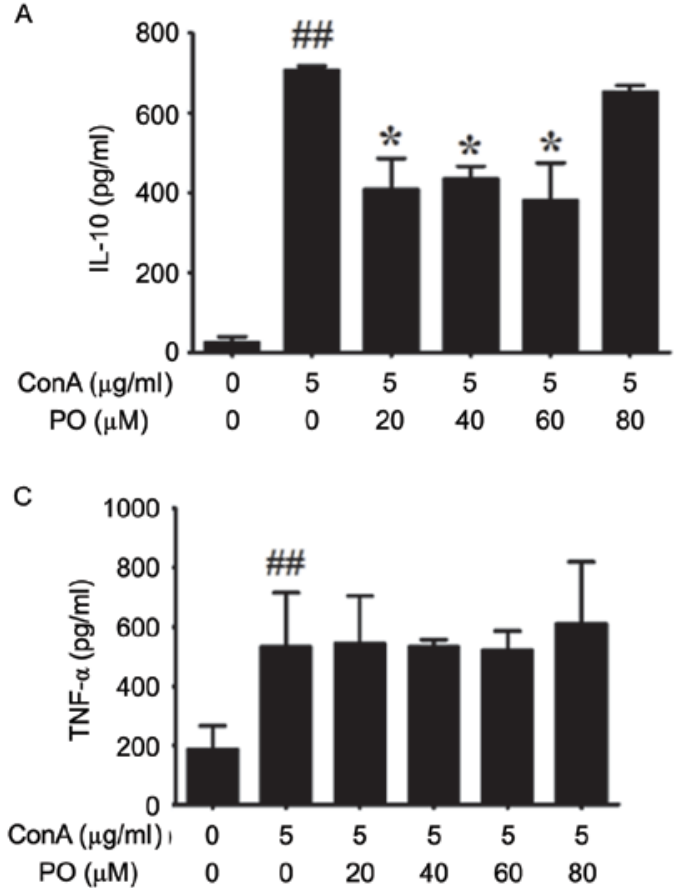

$\mathrm{E}$

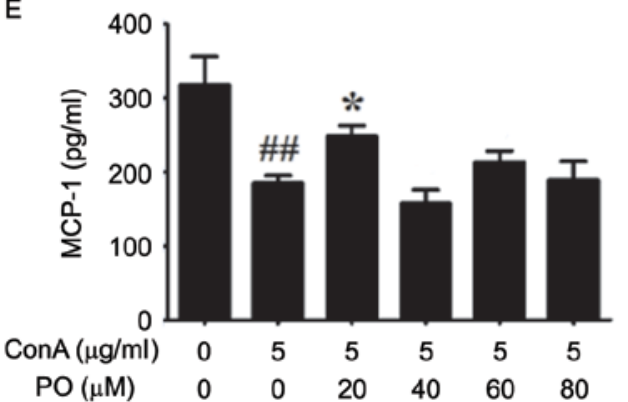

B

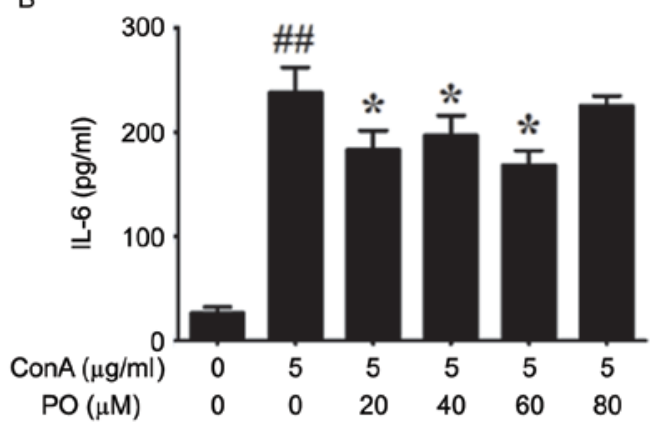

D

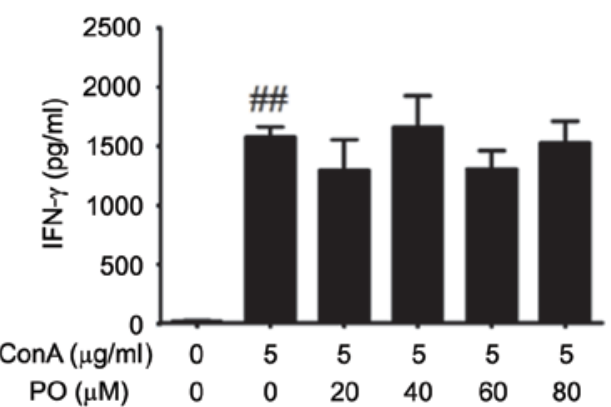

Figure 5. Cytokine profile analysis of the $72 \mathrm{~h}$-ConA-stimulated lymphocytes. (A) IL-10, (B) IL-6, (C) TNF- $\alpha$, (D) IFN- $\gamma$ and (E) MCP-1 were measured in a cytometric bead array. All data were statistically analyzed and displayed in column charts. Values are presented the mean \pm standard deviation $(\mathrm{n}=3)$. ${ }^{\sharp \#} \mathrm{P}<0.01$ vs. control group; "P<0.05 vs. ConA group. IL, interleukin-10; ConA, Concanavalin A; PO, pogostone; TNF- $\alpha$, tumor necrosis factor- $\alpha$; IFN- $\gamma$, interferon- $\gamma$; MCP-1, monocyte chemoattractant protein-1.

on the other hand, IL-10 can promote humoral immune responses, which can results in the development of systemic lupus erythematosus by enhancing class II MHC expression on $\mathrm{B}$ cells and inducing immunoglobulin production (30). TNF is a monocyte-derived family of cytotoxins that have been implicated in tumor regression, septic shock and cachexia, and act as inflammation amplifiers in certain disease, such as Crohn's Disease (31). MCP-1, also referred to as chemokine (C-C motif) ligand 2 , is a recruiter of monocytes, memory $\mathrm{T}$ cells and dendritic cells to the sites of inflammation following tissue injury or infection (32). Therefore, more details were investigated in the present study by the analysis of the inflammatory cytokine profile to determine how PO affected the function of T cells. It was demonstrated that PO decreased the levels of the anti-inflammatory cytokine IL-10, and the pro-inflammatory cytokine IL-6, while the levels of IFN- $\gamma$, MCP-1 and TNF were not affected. Furthermore, a DTH model was used to confirm the immunosuppressive activity of $\mathrm{PO}$ in vivo. DTH is a $\mathrm{T}$ cell-mediated immune response, which is the basis for various immune diseases, including allergic contact dermatitis, autoimmune myocarditis and inflammatory bowel diseases.
As demonstrated by the data of the present study PO relieved the DNCB-induced DTH response by ameliorating the tissue hypertrophy and inflammatory cell infiltration, although only the highest PO dose resulted in a significant reduction of the ear swelling. Together with data from the in vitro experiments, it is rational to propose that direct proliferation inhibition of $\mathrm{PO}$ on the whole population of $\mathrm{T}$ cell may have resulted in changes to the cytokine profile, thus ameliorating the hyperactivity of T cells, such as in the DTH response.

In the current study, experimental evidence demonstrated the immunosuppressive potential of PO, which was mediated by inhibition of $\mathrm{T}$ cell proliferation associated with the suppression of the inflammatory cytokine profile. The DTH model provided further validation of the direct immunosuppressive activity of PO. Compared with the regents with direct cytotoxic activity against T lymphocytes (such as methylprednisolone and azathioprine), our data suggested that the immunosuppressive activity of PO may be a consequence of a 'passivation' of the immune network, rather than from a destruction, as has been manifested by the fact that the proliferation blocking ability of PO was not associated with cytotoxicity in normal lymphocytes or apoptosis 


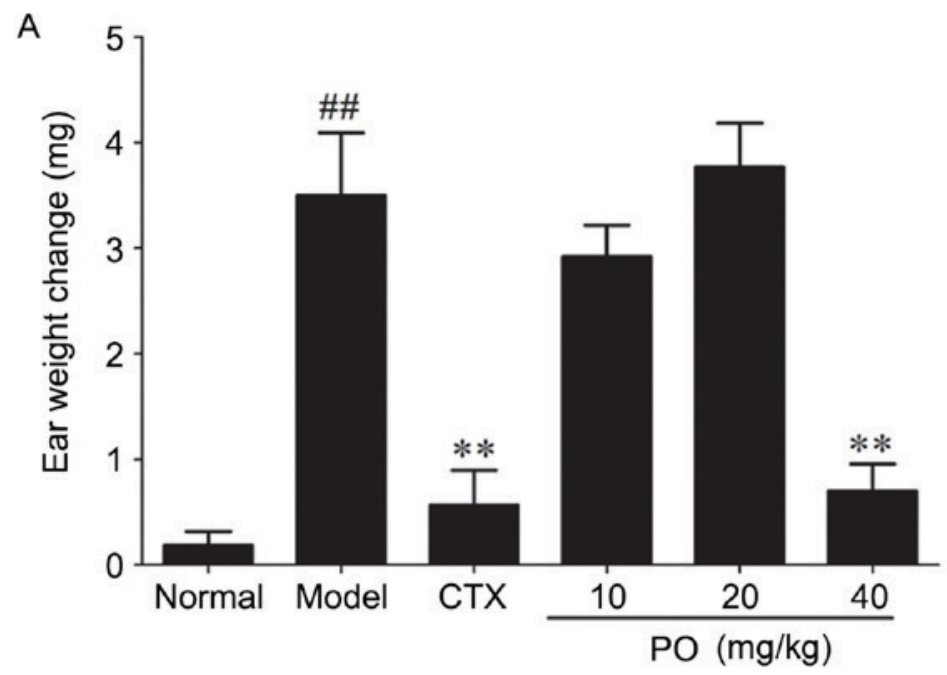

B

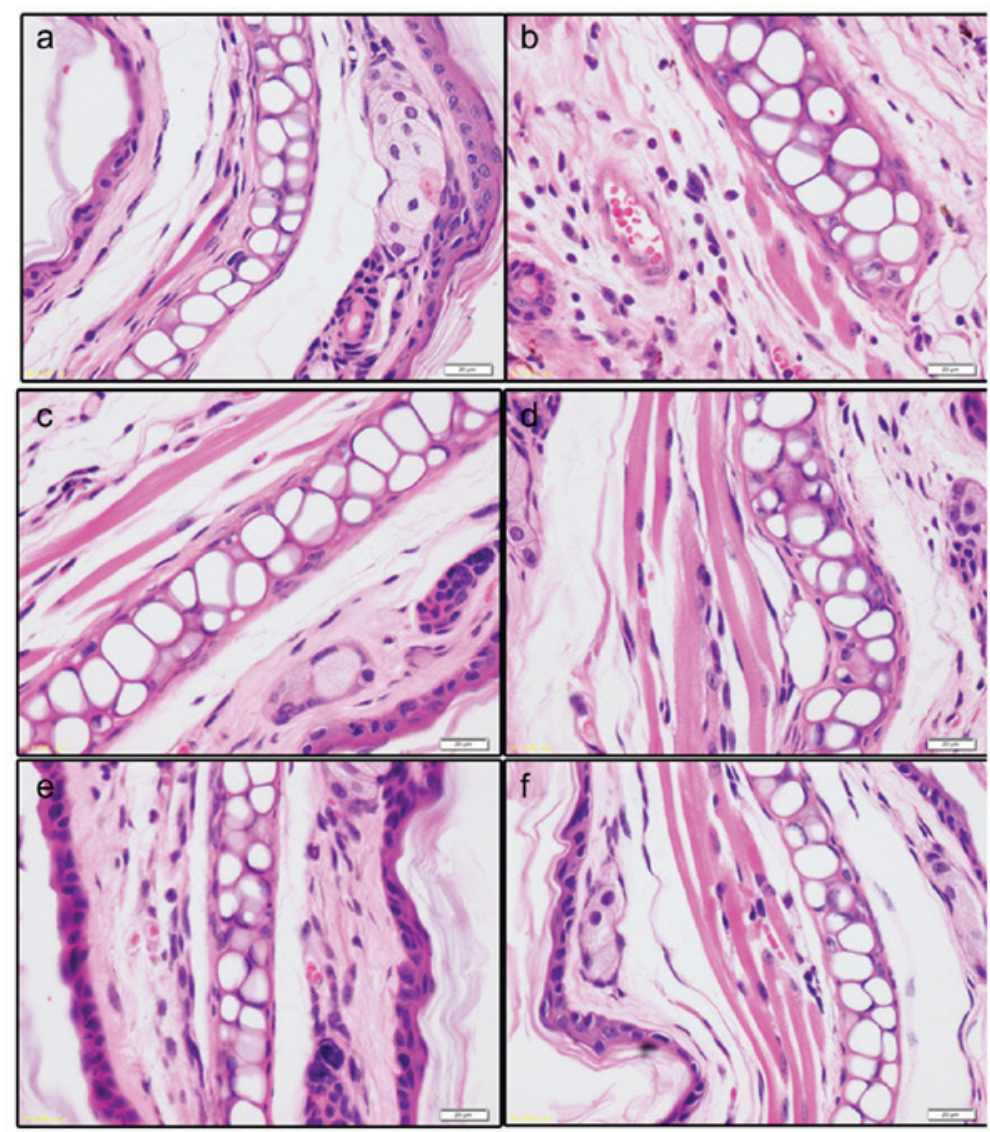

Figure 6. Effects of PO on DNCB-induced delayed type hypersensitivity reaction in Balb/c mice. (A) The ear weight change (mg) was calculated as the difference between the weights of untreated and DNCB-treated ear punches $24 \mathrm{~h}$ after challenge. (B) Representative histology pictures of the DNCB-elicited left ear (magnification, x400). a, Control group; b, Model group; c, CTX group; d, PO $10 \mathrm{mg} / \mathrm{kg}$; e, PO $20 \mathrm{mg} / \mathrm{kg}$; and f, PO $40 \mathrm{mg} / \mathrm{kg}$ groups. Scale bar, $20 \mu \mathrm{m}$. The values are presented as the mean \pm standard deviation $(\mathrm{n}=6)$. ${ }^{\# \#} \mathrm{P}<0.01$ vs. control group; ${ }^{*} \mathrm{P}<0.05$ and ${ }^{* *} \mathrm{P}<0.01$ vs. model group. DNCB, dinitrochlorobenzene; CTX, cyclophosphamide; PO, pogostone.

in ConA-stimulated lymphocytes. Natural products have always been considered as promising immunosuppressant alternatives. It has been previously reported that ginsenoside-Rd (33), alpinetin (34), farrerol (35) and others exhibit immunosuppressive activity through effects of signaling pathways involving nuclear factor of activated T-cells, nuclear factor- $\kappa \mathrm{B}$ and activator protein-1, which are all critical elements for $\mathrm{T}$ cell function. Therefore, determining the potential effects of PO on these signaling pathways will be the task for future research to understand how PO interferes with the differentiation and polarization of $\mathrm{T}$ cell during the excessive immune responses.

\section{Acknowledgements}

This study was financially supported by grants from the National Natural Science Foundation of China (grant 
no. 81403169) and Guangdong Natural Science Foundation (grant no. 2014A030310224), Macao and Taiwan Science \& Technology Cooperation Program of China (grant no. 2014DFH30010), Guangdong Province Universities and Colleges Pearl River Scholar Funded Scheme (2011), and Guangdong Science and Technology Planning Projects (grant nos. 2014A020221023 and 2013B090800052).

\section{References}

1. Chaudhry MS, Velardi E, Dudakov JA and van den Brink MR: Thymus: The next (re)generation. Immunol Rev 271: 56-71, 2016.

2. Farrar CA, Kupiec-Weglinski JW and Sacks SH: The innate immune system and transplantation. Cold Spring Harb Perspect Med 3: a015479, 2013.

3. Leffler J, Bengtsson AA and Blom AM: The complement system in systemic lupus erythematosus: An update. Ann Rheum Dis 73: 1601-1606, 2014.

4. Monteleone G, Caruso R and Pallone F: Targets for new immunomodulation strategies in inflammatory bowel disease. Autoimmun Rev 13: 11-14, 2014.

5. Halloran PF: Immunosuppressive drugs for kidney transplantation. N Engl J Med 351: 2715-2729, 2004.

6. WHO Expert Committee on the Selection Use of Essential Medicines: 19th WHO Model List of Essential Medicines. WHO, Geneva, 2015.

7. Magnasco A, Rossi A, Catarsi P, Gusmano R, Ginevri F, Perfumo F and Ghiggeri GM: Cyclosporin and organ specific toxicity: Clinical aspects, pharmacogenetics and perspectives Curr Clin Pharmacol 3: 166-173, 2008.

8. Zaza G, Tomei P, Ria P, Granata S, Boschiero L and Lupo A: Systemic and nonrenal adverse effects occurring in renal transplant patients treated with mTOR inhibitors. Clin Dev Immunol 2013: 403280, 2013.

9. Jantan I, Ahmad W and Bukhari SN: Plant-derived immunomodulators: An insight on their preclinical evaluation and clinical trials. Front Plant Sci 6: 655, 2015.

10. Lu TC, Liao JC, Huang TH, Lin YC, Liu CY, Chiu YJ and Peng WH: Analgesic and anti-inflammatory activities of the methanol extract from Pogostemon cablin. Evid Based Complement Alternat Med 2011: 671741, 2011.

11. Qi SS, Hu LP, Chen WN, Sun HB and Ma XD: Immunological regulation effects of essential oil in leaves of Cablin Patchouli herbal on mice. Chin Arch Tradit Chin Med 27: 774-776, 2009.

12. Yang Y, Kinoshita K, Koyama K, Takahashi K, Tai T, Nunoura Y and Watanabe K: Anti-emetic principles of Pogostemon cablin (Blanco) Benth. Phytomedicine 6: 89-93, 1999.

13. Li YC, Liang HC, Chen HM, Tan LR, Yi YY, Qin Z, Zhang WM, Wu DW, Li CW, Lin RF, et al: Anti-Candida albicans activity and pharmacokinetics of pogostone isolated from Pogostemonis Herba. Phytomedicine 20:77-83, 2012.

14. Yi YY, He JJ, Su JQ, Kong SZ, Su JY, Li YC, Huang SH, Li CW, Lai XP and Su ZR: Synthesis and antimicrobial evaluation of pogostone and its analogues. Fitoterapia 84: 135-139, 2013.

15. National Research Council: Guide for the care and use of laboratory animals. National Academies Press, Washington (DC), 2011.

16. Yang ZX and Xie PS: Isolation and structure verification of pogostone, an antifungal component from Pogostemon cablin. Chin Sci Bull: 318-320, 1997.
17. Chen HM, Yi YY, Peng SZ, et al: Determination of pogostone in Pogostemon cablin (Blanco) Benth. By HPLC. J Guangzhou Univ Tradit Chin Med 28: 645-647, 2011.

18. Bretscher PA: The activation and inactivation of mature CD4 T cells: A case for peripheral self-nonself discrimination. Scand J Immunol 79: 348-360, 2014.

19. Cantorna MT and Waddell A: The vitamin D receptor turns off chronically activated T cells. Ann N Y Acad Sci 1317: 70-75, 2014.

20. Lee RA and Gabardi S: Current trends in immunosuppressive therapies for renal transplant recipients. Am J Health Syst Pharm 69: 1961-1975, 2012.

21. Dwyer JM and Johnson C: The use of concanavalin A to study the immunoregulation of human T cells. Clin Exp Immunol 46: 237-249, 1981.

22. Atreya I, Diall A, Dvorsky R, Atreya R, Henninger C, Grün M, Hofmann U, Schaeffeler E, López-Posadas R, Daehn I, et al: Designer thiopurine-analogues for optimised immunosuppression in inflammatory bowel diseases. J Crohns Colitis 10: 1132-1143, 2016.

23. Cambiaggi C, Scupoli MT, Cestari T, Gerosa F, Carra G, Tridente G and Accolla RS: Constitutive expression of CD69 in interspecies T-cell hybrids and locus assignment to human chromosome 12. Immunogenetics 36: 117-120, 1992.

24. Imada K and Leonard WJ: The Jak-STAT pathway. Mol Immunol 37: 1-11, 2000.

25. Gonzalez-Rey E, Chorny A and Delgado M: Regulation of immune tolerance by anti-inflammatory neuropeptides. Nat Rev Immunol 7: 52-63, 2007.

26. De Andrea M, Ravera R, Gioia D, Gariglio M and Landolfo S: The interferon system: An overview. Eur J Paediatr Neurol 6 (Suppl A): A41-A46; discussion A55-A58, 2002.

27. Schoenborn JR and Wilson CB: Regulation of interferon-gamma during innate and adaptive immune responses. Adv Immunol 96: 41-101, 2007.

28. Tanaka T, Narazaki $M$ and Kishimoto T: IL-6 in inflammation, immunity, and disease. Cold Spring Harb Perspect Biol 6: a016295, 2014.

29. Minciullo PL, Catalano A, Mandraffino G, Casciaro M, Crucitti A, Maltese G, Morabito N, Lasco A, Gangemi S and Basile G: Inflammaging and anti-inflammaging: The role of cytokines in extreme longevity. Arch Immunol Ther Exp (Warsz) 64: 111-126, 2016

30. Saxena A, Khosraviani S, Noel S, Mohan D, Donner T and Hamad AR: Interleukin-10 paradox: A potent immunoregulatory cytokine that has been difficult to harness for immunotherapy. Cytokine 74: 27-34, 2015.

31. Levin AD, Wildenberg ME and van den Brink GR: Mechanism of action of Anti-TNF therapy in inflammatory bowel disease. J Crohns Colitis 10: 989-997, 2016.

32. Carr MW, Roth SJ, Luther E, Rose SS and Springer TA: Monocyte chemoattractant protein 1 acts as a T-lymphocyte chemoattractant. Proc Natl Acad Sci USA 91: 3652-3656, 1994.

33. Wang L, Zhang Y, Chen J, Li S, Wang Y, Hu L, Wang L and $\mathrm{Wu} \mathrm{Y}$ : Immunosuppressive effects of ginsenoside-Rd on skin allograft rejection in rats. J Surg Res 176: 267-274, 2012.

34. Guan S, Fang B, Song B, Xiong Y and Lu J: Immunosuppressive activity of alpinetin on activation and cytokines secretion of murine T lymphocytes. Immunopharmacol Immunotoxicol 36: 290-296, 2014

35. Xiong Y, Zhang S, Lu J, Sun S, Song B, Xu L, Yang Z and Guan S: Investigation of effects of farrerol on suppression of murine $\mathrm{T}$ lymphocyte activation in vitro and in vivo. Int Immunopharmacol 16: 313-321, 2013. 